\title{
Fitobentos, fitoplancton y zooplancton litoral del bañado de Carilauquen, Cuenca de Llancanelo, Mendoza, Argentina
}

\author{
Patricia Peralta y Verónica Fuentes
}

\author{
Av. Ruiz Leal s/n. C.C.330. IANIGLA. CRICYT. Mendoza (CP 5539) ARGENTINA \\ pperalta@irrigacion.gov.ar/vfuentes@awibremerhaven.de
}

\begin{abstract}
RESUMEN
El ecosistema en estudio constituye uno de los bañados más importantes del humedal de Llancanelo, Provincia de Mendoza, centro-oeste de Argentina.

El objetivo del trabajo fue analizar la calidad del agua basándose en las comunidades planctónicas y bentónicas y la detección del posible impacto de la actividad petrolera sobre las mismas. La investigación se llevó a cabo estacionalmente desde el verano 2001 a primavera de 2002. Las aguas del bañado presentaron altas concentraciones de sulfatos y carbonatos. Se observó un gradiente en la conductividad desde las nacientes a la desembocadura $\left(959 \mu \mathrm{S} \mathrm{cm} \mathrm{cm}^{-1}-10810 \mu \mathrm{S} \mathrm{cm}^{-1}\right)$ lo que determinó un patrón de distribución espacial y estacional de las comunidades analizadas. Entre los grupos algales presentes las diatomeas pennadas fueron dominantes y con mayor abundancia y diversidad en la fracción bentónica. En cuanto al zooplancton, las amebas tecadas fueron el grupo proporcionalmente más abundante, seguidos por rotíferos, ostrácodos y ciliados. Según los requerimientos ambientales de las especies halladas, se define al bañado como eutrófico y polisapróbico caracterizado por la carga elevada de materia orgánica. En las temporadas de invierno y primavera se detectó hidrocarburo en el agua en dos de los sitios de muestreo, por lo que se deduce que esta fuente contaminante podría ser la causa de la marcada disminución de la diversidad planctónica - bentónica y proliferación de algas cianófitas conocidas como tóxicas.
\end{abstract}

Palabras clave: humedal, bañado, fitoplancton, fitobentos, zooplancton

\begin{abstract}
The ecosystem under study represents one of the most important marshes in the Llancanelo wetlands, Mendoza Province, central western Argentina.

The objective of this study was to analyse the planktonic and benthic communities as a measure of water quality, and the detection of the possible impact of oil-production on these communities. The research was carried out seasonally from summer 2001 to spring 2002. The marsh waters presented high concentrations of sulphates and carbonates. A conductivity gradient from the springs to the lake was observed $\left(959 \mu \mathrm{S} \mathrm{cm}^{-1}-10810 \mu \mathrm{S} \mathrm{cm}^{-1}\right)$ which indicated a pattern of spatial and seasonal distribution of the studied communities. Diatoms dominated the algal groups and were the most abundant and diverse in the benthic fraccion. Regarding the zooplankton, shelled amoebas were the most abundant in the zooplankton community, followed by rotifers, ostracods, and ciliates. According to the environmental requirements of the species found, this marsh is defined as eutrophic and polysaprobic, characterized by a high organic matter load. Hydrocarbons were detected in the water in two sampling areas in the winter and spring seasons. Therefore it is hypothesised that this pollutant may be the cause of the marked reduction in planktonic - benthic diversity and the proliferation of cyanophyte algae known to be toxic.
\end{abstract}

Keywords: wetland, marsh, phytoplankton, phytobenthos, zooplankton

\section{INTRODUCCIÓN}

Para muchos grupos taxonómicos los ecosistemas áridos son lugares ricos en endemismos, con especies o grupos de especies de distribución res- tringida a estas áreas, muchas de las cuales sólo ocupan microhábitats específicos. Por otra parte, siendo el agua un importante factor que limita la vida en las zonas áridas, los humedales tanto temporarios como permanentes, congregan innu- 
merables organismos que dependen al menos en algún momento de su ciclo de vida de las condiciones allí presentes (Blendinguer, 2001).

La Laguna de Llancanelo y los bañados circundantes, a pesar de ser un importante escenario para nidificación y alimentación de numerosas especies de aves acuáticas (74) no han sido objeto de estudios limnológicos previos. Las comunidades acuáticas presentes en estos refugios de biodivesidad de ambientes desérticos, se encuentran sujetas a condiciones extremas, principalmente debido a las características climáticas (gran amplitud térmica diaria y estacional). A ello se suma la presión que ejercen las actividades humanas, entre las que se destacan en el caso del Bañado de Carilauquen, la industria petrolera y ganadera.

Este trabajo fue realizado en el marco del "Estudio de Base Cero del Bañado de Carilauquen" por solicitud de la empresa Repsol YPF, como requisito para obtener la autorización gubernamental para la ejecución de pozos de exploración. En el estudio de base se incluyeron además, los estudios de vegetación acuática, macroinvertebrados, aves, mamíferos, reptiles y anfibios.

El presente estudio tiene como objetivo analizar la estructura y dinámica de las comunidades planctónicas y bentónicas del Bañado de Carilauquen, su relación con los parámetros fisico-quimicos y características ecológicas, teniendo en cuanta la influencia de las actividades humanas citadas.

\section{ÁREA DE ESTUdIO}

La laguna de Llancanelo se encuentra localizada en la Provincia de Mendoza, Departamento de Malargüe, en el centro - oeste de la República Argentina, a los $35^{\circ} 45^{\prime} \mathrm{S}$ y $69^{\circ} 08^{\prime} \mathrm{O}$, al pie de las montañas de la Región Andina Central, (1300 m.s.n.m.), (Iglesias y Pérez, 1998). Constituye un humedal salino en una cuenca endorreica, abarcando un área de $65000 \mathrm{ha}$, con $120 \mathrm{~km}$ perimetrales de costa y ambientes acuáticos circundantes, principalmente bañados y pajonales inundables (Sosa et al., 1989), (Fig. 1). A partir del 8 de noviembre de 1995 fue declarada sitio Ramsar.

El Bañado de Carilauquen, posee sus nacientes en los afloramientos de napas subterráneas; recorre $10 \mathrm{Km}$. hasta su desembocadura en la margen centro-oeste de la Laguna de Llancanelo. La profundidad máxima de 2 metros se presenta en las nacientes, reduciéndose hasta $20 \mathrm{~cm}$ en gran parte del bañado. Aisladamente, pueden encontrarse pozones de hasta $1 \mathrm{o} 1.5 \mathrm{~m}$ de profundidad. Presenta terrenos anegados con suelo de tipo limo - arcilloso, con Scirpus californicus, Cortaderia selloana y Chara vulgaris como vegetación dominante.

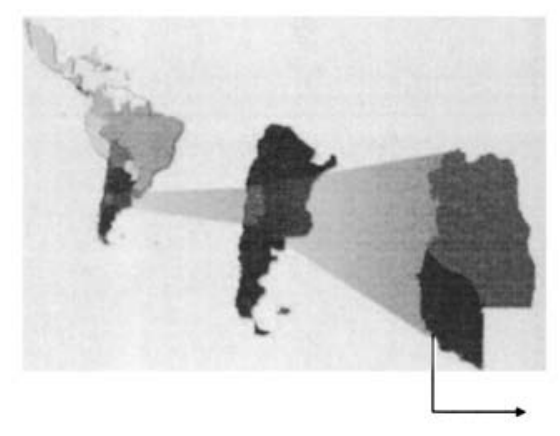

$N$
$\mathbb{N}$
$\mathrm{S}$

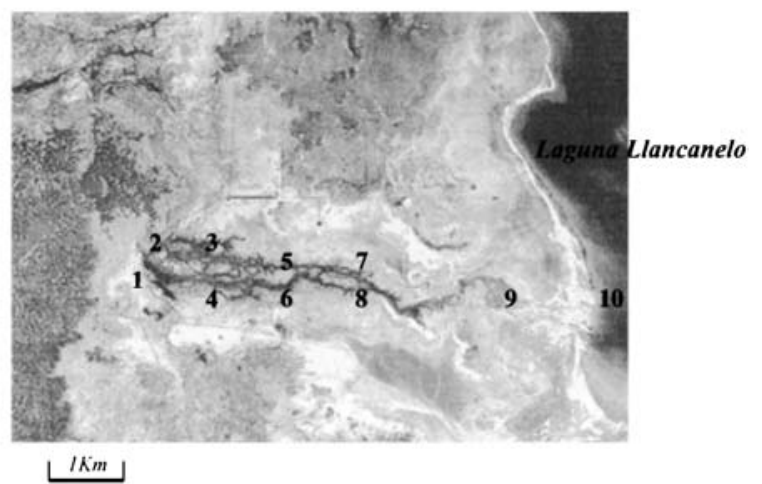

Figura 1. Mapa de ubicación. 1) Nac 1; 2) Nac 2; 3) Up N; 4) Up S; 5) Pozo N; 6) Pozo S; 7) Low N; 8) Low S; 9) Low 2; 10) Low 3. Location map. 1) Nac 1; 2) Nac 2; 3) Up N; 4) Up S; 5) Pozo N; 6) Pozo S; 7) Low N; 8) Low S; 9) Low 2; 10) Low 3. 


\section{MATERIALES Y MÉTODOS}

Se establecieron 10 estaciones de muestreo desde las nacientes a la desembocadura del bañado Se denominaron: Nac 1 y Nac 2 ( las correspondientes a las nacientes del bañado); desde las nacientes hacia aguas abajo los puntos corresponden a ambas márgenes del bañado (N: Norte y S: Sur). Up N y Up S (aguas arriba del sitio donde se perforaría el pozo de exploración petrolera); Pozo N y Pozo S (sitio donde se perforaría el pozo de exploración petrolera); Low N y Low $\mathrm{S}$ (aguas debajo de los pozos); Low 2 (desembocadura del bañado; Low 3 (Laguna de Llancanelo), (Fig.1).

Las muestras de zooplancton fueron extraídas, por duplicado, mediante el filtrado de 50 litros de agua a través de una red de $30 \mu \mathrm{m}$ de abertura de malla. Las mismas fueron fijadas con formol al $4 \%$. Las muestras destinadas a la cualificación del fitoplancton fueron tomadas mediante arrastre de red de $30 \mu \mathrm{m}$ de poro y conservación en formol $4 \%$. Se tomaron por triplicado $100 \mathrm{ml}$ de agua fijada con Lugol, para el análisis cuantitativo (APHA, 1995).

Para el estudio de las comunidades fitobentónicas se tomaron muestras de los primeros 5$10 \mathrm{~mm}$ del sedimento (por triplicado), considerada como la porción fotosintéticamente activa. Siendo la profundidad recomendable para la extracción, la comprendida entre los 10 y $30 \mathrm{~cm}$ de la superficie del agua (Gómez, 1999; Descy \& Coste, 1990). La conservación de las muestras se realizó con formol al $4 \%$ en un recipiente de volumen conocido para la cuantificación.

Los recuentos de zooplancton se realizaron con cámara de Sedwick-Rafter de $1 \mathrm{ml}$ de capacidad, en microscopio binocular, hasta alcanzar un coeficiente de variación de $20 \%$ (APHA, 1995). Los resultados se expresan en número de individuos por litro. El recuento de fitoplancton y fitobentos se realizó en cubetas de sedimentación mediante el uso de microscopio invertido. Los resultados de abundancia se expresan en células por mililitro (cel/ml) para el fitoplancton y células por centímetro cuadrado $\left(\mathrm{cel} / \mathrm{cm}^{2}\right)$ para el fitobentos.

Con el objetivo de determinar características estructurales de las comunidades, se calcularon los índices de diversidad específica de Shannon $\&$ Weaver $\left(\mathrm{H}^{\prime}\right)$ y de Dominancia de Simpson $(\lambda)$ (Brower and Zar, 1977). Asimismo, se tuvieron en cuenta las características autoecológicas de las especies identificadas con el objetivo de identificar especies con requerimientos ambientales particulares, así como determinar el estado trófico y sapróbico del bañado.

Simultáneamente a la toma de muestras se midieron in situ temperatura del ambiente, temperatura del agua, $\mathrm{pH}$ y conductividad. En 5 estaciones (Nac1, Nac2, Low N, Low 2 y Low 3 ) se tomaron muestras destinadas a la medición de parámetros químicos en laboratorio: oxígeno disuelto, nitratos, carbonatos, bicarbonatos, fósforo total, magnesio, sodio, potasio, calcio, sulfatos, cloruros. Metales pesados: arsénico, mercurio, cromo hexavalente, plomo, plata, cobalto, zinc e hidrocarburos parafínicos totales.

\section{RESULTADOS}

\section{Análisis químicos}

En las tablas 1 y 2 se presentan los resultados obtenidos de los parámetros físicos y químicos estimados, respectivamente.

Las aguas del Bañado de Carilauquen son predominantemente sulfatadas cálcicas. Se observa un marcado gradiente de evolución química desde las nacientes hacia la desembocadura. Los valores de conductividad medidos presentaron un promedio de $959 \mu \mathrm{S} \mathrm{cm}^{-1}$ en las nacientes y de $10810 \mu \mathrm{S} \mathrm{cm}^{-1}$ en la desembocadura y laguna de Llancanelo. Este gradiente se debe al incremento en concentración de iones como bicarbonatos, sulfatos, sodio, potasio, calcio, magnesio, cloruros. Considerando la extensión del bañado, esta evolución se atribuye al alto nivel de infiltración y la escasez de precipitaciones en el área, que favorece la evaporación y la modificación de la impronta geoquímica a través de las concentraciones de sales (Ostera, 2001).

Se detectó presencia de hidrocarburos en agua superficial en las estaciones de invierno y 
Tabla 1. Parámetros físico-químicos (I) obtenidos en el Bañado de Carilauquen. Physical and chemical parameters (I) obtained from the Bañado de Carilauquen.

\begin{tabular}{|c|c|c|c|c|c|c|c|c|c|c|c|c|c|c|c|c|c|c|c|c|}
\hline & \multicolumn{4}{|c|}{ NAC 1} & \multicolumn{4}{|c|}{ NAC 2} & \multicolumn{4}{|c|}{ LOW N } & \multicolumn{3}{|c|}{ LOW 2} & \multicolumn{5}{|c|}{ LOW 3} \\
\hline & Ver & Otoño & Invier & Prim & Ver & Otoño & Invier & Prim & Ver & Otoño & Invier & Prim & Ver & Otoño & Invier & Prim & Ver & Otoño & Invier & Prim \\
\hline $\mathrm{HCO}_{3}^{-} \mathrm{mg} / \mathrm{l}$ & & 183 & 122 & 152.5 & & 244 & 122 & 152.5 & & 170.8 & 122 & 91.5 & & 170 & 91.5 & 91.5 & & 122 & 91.5 & 91.5 \\
\hline Oxíg. & 7.2 & 6.8 & 5.6 & 5.4 & 7.8 & 7 & 5.8 & 5.7 & 7.3 & 6.9 & 6.4 & 5.5 & 7.1 & 6.9 & 6 & 5.3 & 7.9 & 7 & 6.3 & 5.6 \\
\hline \multicolumn{21}{|l|}{ Disuelto (mg/l) } \\
\hline $\mathrm{Cl}^{-}(\mathrm{mg} / \mathrm{l})$ & 11 & 35.5 & 17.7 & 17.75 & 10 & 26.4 & 26.6 & 17.75 & 15 & 71 & 35.4 & 2577.3 & 4352 & 6933.2 & 3105.7 & 2488.6 & 3132 & 4267.1 & 3105.7 & 2399.8 \\
\hline \multirow{2}{*}{ Ptotal } & $<0.2$ & $<1$ & & & $<0.2$ & $<1$ & & & $<0.2$ & $<1$ & & & $<0.2$ & $<1$ & & & $<0.2$ & $<1$ & & \\
\hline & $\mathrm{mg} / 1$ & ppm & & & $\mathrm{mg} / 1$ & ppm & & & $\mathrm{mg} / \mathrm{l}$ & ppm & & & $\mathrm{mg} / \mathrm{l}$ & ppm & & & $\mathrm{mg} / \mathrm{l}$ & ppm & & \\
\hline $\mathrm{SO} 4{ }^{2-(m g / l)}$ & 465 & 340.8 & 384 & 350.4 & 411 & 278.4 & 384.2 & 393.6 & 650 & 724 & 509.1 & 1080 & 2747 & 1833.6 & 1580.2 & 1185.6 & 2255 & 2068.8 & 1580.2 & 1406.4 \\
\hline $\mathrm{N}-\mathrm{NO}_{3}^{-}$ & $<0.2$ & 0.70 & & 0.43 & $<0.2$ & 0.60 & & 1.1 & $<0.2$ & ND & & $\mathrm{ND}$ & $<0.2$ & ND & & ND & $<0.2$ & ND & & ND \\
\hline $\mathrm{Na}$ & 23.9 & 16.10 & 13.8 & 13.8 & 19.4 & 13.8 & 16.1 & 13.8 & 32 & 92 & 20.7 & 25.3 & 2600 & 3307.4 & 1361 & 995.9 & 1900 & 2270.1 & 1499 & 991.3 \\
\hline $\mathrm{K}^{+}$ & 3 & ND & 3.9 & 2.34 & 2.8 & ND & 3.9 & 3.12 & 3.5 & 7.8 & 7.8 & 7.8 & 103 & 159.9 & 97.7 & 81.9 & 77 & 117 & 101.7 & 81.9 \\
\hline $\mathrm{Ca}^{+}$ & 152 & 178 & 192.4 & 180 & 154 & 178 & 184.4 & 200 & 242 & 299 & 216.4 & 236 & 1290 & 944 & 841.3 & 680 & 1220 & 748 & 737.5 & 710 \\
\hline $\mathrm{Mg}^{+}$ & 6.3 & 7.2 & 7.7 & 8.4 & 6.3 & 7.2 & 7.3 & 8.4 & 9.1 & 12 & 12.2 & 14.4 & 202 & 226.8 & 145.9 & 99.6 & 135 & 178.8 & 145.7 & 102 \\
\hline $\begin{array}{l}\text { Hidrocarburo } \\
(\mathrm{mg} / \mathrm{l})\end{array}$ & ND & ND & ND & ND & ND & $\mathrm{ND}$ & ND & ND & ND & ND & 11.2 & 2.8 & ND & ND & 1.8 & ND & ND & ND & ND & ND \\
\hline Metales pesados & ND & ND & ND & ND & ND & ND & ND & ND & ND & ND & ND & ND & ND & ND & ND & ND & ND & ND & ND & ND \\
\hline
\end{tabular}

primavera. En invierno en el sitio denominado Low N con $11.2 \mathrm{mg} / \mathrm{l}$; en primavera $2.8 \mathrm{mg} / 1$.

En el punto de muestreo correspondiente a la desembocadura del bañado (Low 2) se detectó hidrocarburo en invierno con una concentración de $1.8 \mathrm{mg} / 1$.

En ninguna de las temporadas analizadas se detectaron metales pesados en el agua.

\section{Fitoplancton}

En total se identificaron 136 especies de algas correspondiendo a 97 especies de diatomeas, 17 cianofitas, 17 clorofitas, 2 euglenofitas, 2 dinoflagelados y 1 especie de xantoficea.

Muchas de las algas identificadas se presentaron en todas las estaciones del año, mientras que otras presentaron marcada estacionalidad. Entre estas últimas se identifican como representativas del verano a: Cymbella cistula, C. tumida, Cocconeis pseudomarginata, Epithemia argus, E. sorex, Gomphonema capitatum, Gyrosigma acuminatum, Hannea arcus, Navicula cuspidata var. ambigua, $N$. radiosa, $N$. sigmoidea, $N$. conmutata, $N$. dubia, Surirella biseriata, S. linearis, $S$. tabulata, Spirulina subsalsa, Gymnodinium sp., Microspora crassior y M. tumidula. Otras fueron exclusivas del otoño como Gomphonema elongatum, Gyrosigma balticum, Hantzchia sp., Nitzschia romana, N. scalaris, Aphanocapsa rivularis, Lyngbya birgei, Merismopedia glauca, Tolypothrix tenuis y Botryochloris minima (Tabla 3).

Tabla 2. Parámetros físico-químicos (II) obtenidos en el Bañado de Carilauquen. Physical and chemical parameters obtained from the Bañado de Carilauquen.

\begin{tabular}{lcccccccccc}
\hline & NAC1 & NAC2 & UPS & UPN & POZO N & POZO S & LOWS & LOWN & LOW2 & LOW3 \\
\hline Cond. $\left(\mu \mathrm{S} \mathrm{cm}^{-1}\right)$ & 957.25 & 975.75 & 1476.5 & 1129 & 1250.5 & 1097.5 & 1264 & 1297.25 & 11630 & 10810 \\
$\mathrm{~T}^{\mathrm{o}}$ agua & 12.975 & 13.75 & 14.225 & 14.1 & 13.2 & 11.85 & 13.875 & 13.25 & 13.4 & 11.725 \\
$\mathrm{pH}$ & 7.6 & 7.5 & 8 & 8 & 7.38 & 7.5 & 7.4475 & 7.69 & 7.1125 & 7.235 \\
\hline
\end{tabular}


Tabla 3. Especies de fitoplancton determinadas en el Bañado de Carilauquen. Phytoplankton species determined in the Bañado de Carilauquen.

\begin{tabular}{|c|c|c|c|c|}
\hline & VERANO & OTOÑO & INVIERNO & PRIMAVERA \\
\hline Achnantes minuttissima Raben. & $\mathrm{X}$ & $\mathrm{X}$ & $\mathrm{X}$ & $\mathrm{X}$ \\
\hline Achnantes sp 1 & $\mathrm{X}$ & $\mathrm{X}$ & $\mathrm{X}$ & $\mathrm{X}$ \\
\hline Amphiprora angustatumHendley & & $\mathrm{X}$ & $\mathrm{X}$ & \\
\hline Amphora coffeiformis Agardh & $\mathrm{X}$ & $\mathrm{X}$ & $\mathrm{X}$ & $\mathrm{X}$ \\
\hline Amphora lineolata Ehr. Kramer & $\mathrm{X}$ & $\mathrm{X}$ & $\mathrm{X}$ & $\mathrm{X}$ \\
\hline Amphora ovalis Kütz & $\mathrm{X}$ & $\mathrm{X}$ & $\mathrm{X}$ & $\mathrm{X}$ \\
\hline Amphora proteus Kütz & $\mathrm{X}$ & $\mathrm{X}$ & $\mathrm{X}$ & \\
\hline Amphora veneta Kütz & $\mathrm{X}$ & $\mathrm{X}$ & $\mathrm{X}$ & $\mathrm{X}$ \\
\hline Anomoneis sphaerophora (Ehr)Muller & & & $\mathrm{X}$ & \\
\hline Asterionella formosa Hass & & $\mathrm{X}$ & $\mathrm{X}$ & $\mathrm{X}$ \\
\hline Campylodiscus clypeus Ehr. & $\mathrm{X}$ & $\mathrm{X}$ & $\mathrm{X}$ & $\mathrm{X}$ \\
\hline Campylodiscus noricus Ehr. & & $\mathrm{X}$ & $\mathrm{X}$ & $\mathrm{X}$ \\
\hline Cocconeis placentula Ehr & $\mathrm{X}$ & $\mathrm{X}$ & $\mathrm{X}$ & $\mathrm{X}$ \\
\hline Cyclotella meneghiniana Kützing & & & $\mathrm{X}$ & $\mathrm{X}$ \\
\hline Cymbela cistula (Hemprich) & $\mathrm{X}$ & & & \\
\hline Cymbela minutaHilse ex Rabenhors & $\mathrm{X}$ & $\mathrm{X}$ & $\mathrm{X}$ & $\mathrm{X}$ \\
\hline Cymbela muellerif. ventricosa Reim & $\mathrm{X}$ & $\mathrm{X}$ & & \\
\hline Cymbella pusilla Grun & & $\mathrm{X}$ & & \\
\hline Cymbela tumida (de Brebisson) & $\mathrm{X}$ & & & \\
\hline Cymbela tumidula Grun. & & $\mathrm{X}$ & & \\
\hline Cymbela ventricosa Kütz & $\mathrm{X}$ & $\mathrm{X}$ & $\mathrm{X}$ & $\mathrm{X}$ \\
\hline Denticula elegans (Kütz) & $\mathrm{X}$ & $\mathrm{X}$ & $\mathrm{X}$ & $\mathrm{X}$ \\
\hline Diatoma vulgare Bory & $\mathrm{X}$ & $\mathrm{X}$ & $\mathrm{X}$ & $\mathrm{X}$ \\
\hline Diploneis elliptica Kütz & & $\mathrm{X}$ & $\mathrm{X}$ & $\mathrm{X}$ \\
\hline Diploneis smithii (de Brebisson) & $\mathrm{X}$ & $\mathrm{X}$ & $\mathrm{X}$ & \\
\hline Ephitemia adnata & & & & $\mathrm{X}$ \\
\hline Ephitemia argus Kütz & $\mathrm{X}$ & & & \\
\hline Ephitemia sorex Kütz & $\mathrm{X}$ & & & \\
\hline Epithemia turgida (Ehr.) Kütz. & & $\mathrm{X}$ & $\mathrm{X}$ & $\mathrm{X}$ \\
\hline Epithemia zebra (Ehr.) Kütz & $\mathrm{X}$ & $\mathrm{X}$ & $\mathrm{X}$ & $\mathrm{X}$ \\
\hline Eunotia pectinalis var. minor (Kütz) Rabh. & $\mathrm{X}$ & $\mathrm{X}$ & $\mathrm{X}$ & $\mathrm{X}$ \\
\hline Fragilaria ulna (Nietz.) Ehr. & $\mathrm{X}$ & $\mathrm{X}$ & $\mathrm{X}$ & $\mathrm{X}$ \\
\hline Fragilaria virescens Ralfs & $\mathrm{X}$ & $\mathrm{X}$ & $\mathrm{X}$ & $\mathrm{X}$ \\
\hline Gomphonema acuminatum Ehr. & $\mathrm{X}$ & $\mathrm{X}$ & $\mathrm{X}$ & $\mathrm{X}$ \\
\hline Gomphonema angustatum Kütz. & & $\mathrm{X}$ & & \\
\hline Gomphonema capitatum & $\mathrm{X}$ & & & \\
\hline Gomphonema constrictum Ehr. & $\mathrm{X}$ & $\mathrm{X}$ & & \\
\hline Gomphonerma elongatum & & $\mathrm{X}$ & & \\
\hline Gomphonema lanceolatum Ehr & & $\mathrm{X}$ & $\mathrm{X}$ & $\mathrm{X}$ \\
\hline Gomphonema parvulum Kütz. & & $\mathrm{X}$ & & \\
\hline Gomphonema truncatum Ehr. & & $\mathrm{X}$ & & \\
\hline Gyrosigma acuminatum Kütz & $\mathrm{X}$ & & & \\
\hline Gyrosigma balticum Ehr. & & $\mathrm{X}$ & & \\
\hline Gyrosigma spenceri (Quekett)Griffith & & $\mathrm{X}$ & & \\
\hline Hantzschia sp. & & $\mathrm{X}$ & & \\
\hline Hannaea arcusEhr. & $\mathrm{X}$ & & & \\
\hline Hyalodiscus laevis Ehr & $\mathrm{X}$ & $\mathrm{X}$ & & \\
\hline Mastoglia braunii (Grunow) & $\mathrm{X}$ & $\mathrm{X}$ & $\mathrm{X}$ & $\mathrm{X}$ \\
\hline Mastoglia elliptica (Agardh) Cleve & $\mathrm{X}$ & $\mathrm{X}$ & $\mathrm{X}$ & $\mathrm{X}$ \\
\hline Mastoglia ovalis & & & & $\mathrm{X}$ \\
\hline
\end{tabular}


Tabla 3. Continuación. Continuation.

\begin{tabular}{|c|c|c|c|c|}
\hline & VERANO & OTOÑO & INVIERNO & PRIMAVERA \\
\hline Mastoglia smithii Thwaites & $\mathrm{X}$ & $\mathrm{X}$ & $\mathrm{X}$ & $\mathrm{X}$ \\
\hline Melosira nummulö̈des (Dilleu) Agardh & $\mathrm{X}$ & $\mathrm{X}$ & $\mathrm{X}$ & $\mathrm{X}$ \\
\hline Melosira varians Agardh & $\mathrm{X}$ & $\mathrm{X}$ & $\mathrm{X}$ & $\mathrm{X}$ \\
\hline Navicula cryptocephala Kütz & $\mathrm{X}$ & $\mathrm{X}$ & & \\
\hline Navicula cincta (Ehr) & $\mathrm{X}$ & $\mathrm{X}$ & $\mathrm{X}$ & $\mathrm{X}$ \\
\hline Navicula cuspidata Kütz & $\mathrm{X}$ & $\mathrm{X}$ & $\mathrm{X}$ & $\mathrm{X}$ \\
\hline N.cuspidata var ambigua & $\mathrm{X}$ & & & \\
\hline Navicula goppertiana (Bleisch) Grun & & $\mathrm{X}$ & $\mathrm{X}$ & \\
\hline Navicula gregaria Donkin & & $\mathrm{X}$ & & $\mathrm{X}$ \\
\hline Navicula imbricata Bock & & $\mathrm{X}$ & $\mathrm{X}$ & \\
\hline Navicula peregrina Kütz & & $\mathrm{X}$ & & $\mathrm{X}$ \\
\hline Navicula pygmea Ehr. & & $\mathrm{X}$ & $\mathrm{X}$ & $\mathrm{X}$ \\
\hline Navicula pupula Kützing & $\mathrm{X}$ & $\mathrm{X}$ & $\mathrm{X}$ & $\mathrm{X}$ \\
\hline Navicula radiosa (Brebisson) & $\mathrm{X}$ & & & \\
\hline Navicula salinarum Grun & $\mathrm{X}$ & & & $\mathrm{X}$ \\
\hline Navicula soodensis Kraskke & $\mathrm{X}$ & & & $\mathrm{X}$ \\
\hline Nitzschia apiculata (Gregory) Grun. & & $\mathrm{X}$ & $\mathrm{X}$ & \\
\hline Nitzschia conmutata Grun & $\mathrm{X}$ & & & \\
\hline Nitzschia constricta & $\mathrm{X}$ & & $\mathrm{X}$ & $\mathrm{X}$ \\
\hline Nitzschia dubia W. Smith & $\mathrm{X}$ & & & \\
\hline Nitzchia hustedtiana Salah & & $\mathrm{X}$ & $\mathrm{X}$ & $\mathrm{X}$ \\
\hline Nitzschia linearis & & & $\mathrm{X}$ & $\mathrm{X}$ \\
\hline Nitzschia palea (Kütz) Smith & $\mathrm{X}$ & $\mathrm{X}$ & $\mathrm{X}$ & $\mathrm{X}$ \\
\hline Nitzchia romana Grun & & $\mathrm{X}$ & & \\
\hline Nitzchia scalaris (Ehr) Wismith & & $\mathrm{X}$ & & \\
\hline Nitzchia sigma (Kütz) Smith & & $\mathrm{X}$ & $\mathrm{X}$ & $\mathrm{X}$ \\
\hline Nitzschia sigmoidea (Ehr) Smith & $\mathrm{X}$ & & & \\
\hline Pinnularia gibba Ehr. & $\mathrm{X}$ & $\mathrm{X}$ & $\mathrm{X}$ & $\mathrm{X}$ \\
\hline Pinnularia maior (Kütz) Cleve & $\mathrm{X}$ & $\mathrm{X}$ & $\mathrm{X}$ & $\mathrm{X}$ \\
\hline Rhopalodia gibberula var. heurkii Müller & $\mathrm{X}$ & $\mathrm{X}$ & $\mathrm{X}$ & $\mathrm{X}$ \\
\hline Rhoicosphaenia curvata (Kütz) Grun & $\mathrm{X}$ & $\mathrm{X}$ & $\mathrm{X}$ & $\mathrm{X}$ \\
\hline Rhopalodia gibba (Ehr) Muller & $\mathrm{X}$ & $\mathrm{X}$ & $\mathrm{X}$ & $\mathrm{X}$ \\
\hline Rhopalodia parallela (Grun) Mull. & $\mathrm{X}$ & $\mathrm{X}$ & $\mathrm{X}$ & $\mathrm{X}$ \\
\hline Stauroneis phenicenteron Ehr. & $\mathrm{X}$ & $\mathrm{X}$ & $\mathrm{X}$ & $\mathrm{X}$ \\
\hline Surilella biseriata Brebisson & $\mathrm{X}$ & & & \\
\hline Surirella ovalis de Brebisson & $\mathrm{X}$ & & $\mathrm{X}$ & $\mathrm{X}$ \\
\hline Surilella robusta & & & & $\mathrm{X}$ \\
\hline Surirella striatula Turpin & $\mathrm{X}$ & $\mathrm{X}$ & $\mathrm{X}$ & $\mathrm{X}$ \\
\hline Synedra minuscula Grun & $\mathrm{X}$ & $\mathrm{X}$ & $\mathrm{X}$ & $\mathrm{X}$ \\
\hline Synedra rupens & & & & $\mathrm{X}$ \\
\hline Synedra tabulata Agardh & $\mathrm{X}$ & & & \\
\hline Anabaena inaequalis (Kütz) Bornet y Flab. & $\mathrm{X}$ & $\mathrm{X}$ & $\mathrm{X}$ & $\mathrm{X}$ \\
\hline Anabaena torulosa (Carm) Lagerh. & $\mathrm{X}$ & $\mathrm{X}$ & $\mathrm{X}$ & $\mathrm{X}$ \\
\hline Aphanocapsa rivularis & & $\mathrm{X}$ & & \\
\hline Chroococus turgidus Kütz & $\mathrm{X}$ & $\mathrm{X}$ & & $\mathrm{X}$ \\
\hline Lyngbia birgei Smith & & $\mathrm{X}$ & & $\mathrm{X}$ \\
\hline Lyngbya limnetica Lemm. & $\mathrm{X}$ & $\mathrm{X}$ & $\mathrm{X}$ & $\mathrm{X}$ \\
\hline Merismopedia elegans Braun & & & & $\mathrm{X}$ \\
\hline Merismopedia glauca & $\mathrm{X}$ & & $\mathrm{X}$ & $\mathrm{X}$ \\
\hline Oscillatoria formosa Bory & $\mathrm{X}$ & $\mathrm{X}$ & $\mathrm{X}$ & $\mathrm{X}$ \\
\hline Oscillatoria limosa Agardh & & $\mathrm{X}$ & & $\mathrm{X}$ \\
\hline
\end{tabular}


Tabla 3. Continuación. Continuation.

\begin{tabular}{|c|c|c|c|c|}
\hline & VERANO & OTOÑO & INVIERNO & PRIMAVERA \\
\hline Oscillatoria princeps Vancher & $\mathrm{X}$ & $\mathrm{X}$ & $\mathrm{X}$ & $\mathrm{X}$ \\
\hline Oscilatoria tenuis & & & & $\mathrm{X}$ \\
\hline Pseudoanabaena $\mathrm{sp}$ & & $\mathrm{X}$ & & $\mathrm{X}$ \\
\hline Spirulina princeps (W. Et. West) & $\mathrm{X}$ & $\mathrm{X}$ & & $\mathrm{X}$ \\
\hline Spirulina subsalsa Oesrsted & $\mathrm{X}$ & & & \\
\hline Synechocystis aqualitis Sauv. & & & & $\mathrm{X}$ \\
\hline Toplipothrix tenuis Kütz & & $\mathrm{X}$ & & \\
\hline Ankistrodesmus sp. & & & & $\mathrm{X}$ \\
\hline Botryococcus braunii Kütz & $\mathrm{X}$ & $\mathrm{X}$ & & $\mathrm{X}$ \\
\hline Coelastrum sp. & $\mathrm{X}$ & & & \\
\hline Cosmarium botrytis Menegh. & $\mathrm{X}$ & $\mathrm{X}$ & & $\mathrm{X}$ \\
\hline Cosmarium reniformis (Ralfs) & $\mathrm{X}$ & $\mathrm{X}$ & $\mathrm{X}$ & $\mathrm{X}$ \\
\hline Closteriopsis sp. & $\mathrm{X}$ & $\mathrm{X}$ & & $\mathrm{X}$ \\
\hline Euastrum turgidum Wall & $\mathrm{X}$ & $\mathrm{X}$ & & $\mathrm{X}$ \\
\hline Microspora crassior (Hans) Hazen & $\mathrm{X}$ & & & \\
\hline Microspora tumidula Hazen & $\mathrm{X}$ & & & \\
\hline Oedogonium sp. & & & $\mathrm{X}$ & \\
\hline Scenedesmus sp. & $\mathrm{X}$ & $\mathrm{X}$ & $\mathrm{X}$ & $\mathrm{X}$ \\
\hline \multicolumn{5}{|l|}{ Sphaerocystis schoeteri Chod } \\
\hline Spyrogyra mirabilis (Hassal) Kütz & $\mathrm{X}$ & $\mathrm{X}$ & $\mathrm{X}$ & $\mathrm{X}$ \\
\hline Spyrogyra sp 2 & $\mathrm{X}$ & $\mathrm{X}$ & & \\
\hline Uronema elongatum Hodgetts & $\mathrm{X}$ & $\mathrm{X}$ & $\mathrm{X}$ & $\mathrm{X}$ \\
\hline Zygnema pectinatum (Vauch) Ag. & $\mathrm{X}$ & $\mathrm{X}$ & & $\mathrm{X}$ \\
\hline Gymnodinium sp. & $\mathrm{X}$ & & & \\
\hline Pseudoperidinium & & $\mathrm{X}$ & $\mathrm{X}$ & $\mathrm{X}$ \\
\hline Euglena oxiuris var. charcowiensis & $\mathrm{X}$ & $\mathrm{X}$ & & \\
\hline Euglena proxima & & & $\mathrm{X}$ & $\mathrm{X}$ \\
\hline
\end{tabular}

Se pueden mencionar a diatomeas características de ambientes salobres y marinos como Amphiprora angustatum, Amphora coffeiformis, Campylodiscus clypeus, C. noricus, Diploneis elliptica, Hyalodiscus laevis, Melosira moniliformis y Surirella striatula.

Con respecto al índice de diversidad de Shannon, promedio por temporada, se observó una clara disminución del valor en la temporada de invierno (0.89), siendo el mayor observado en la temporada de verano (1.9), primavera (1.64) y otoño (1.19).

La figura 2 representa el número de especies por temporada y sitio de muestreo, como así también la proporción de grupos algales. En todas las estaciones del año y en cada uno de los sitios de muestreo dominaron las diatomeas siendo subdominantes las clorofitas en verano e invierno; y cianofitas en otoño y primavera.

En la temporada de verano, las estaciones Nac 2 y Low 2 presentaron el mayor número de especies (37). El sitio Nac 2 se caracterizó por presentar mayor riqueza de cianofitas; mientras que en la desembocadura del bañado fueron frecuentes las diatomeas.

En la temporada de otoño las estaciones $\mathrm{Nac}$ 1, Nac 2 y Low 2 presentaron el mayor número de especies, con diatomeas como dominantes y clorofitas y cianofitas como co-dominantes. En la temporada de invierno se observó disminución del número de especies, con alta representatividad de diatomeas, cianofitas y clorofitas. La disminución en el número de especies algales registrada en esta temporada, podría deberse 
al aumento de caudal en el bañado y arrastre hacia a la laguna de Llancanelo, donde se detectó un ligero aumento del número de especies. En la temporada de primavera, las estaciones Nac 2, PozoN y Low N presentaron la mayor riqueza específica, con mayor proporción de clorofitas. La densidad celular (células por mililitro), varió considerablemente no sólo entre las temporadas de estudio, sino también entre los sitios de muestreo (Fig. 2). En las temporadas estivales se registraron las menores densidades, con 2655 y $12710 \mathrm{cel} / \mathrm{ml}$ para verano y primavera, respectivamente. En las temporadas de otoño e invierno se observó un marcado incremento con un promedio de abundancia de $402710 \mathrm{cel} / \mathrm{ml}$ en otoño y $2655320 \mathrm{cel} / \mathrm{ml}$ en invierno. Los picos de abundancia absoluta están dados por las cianofitas Lyngbya limneti-
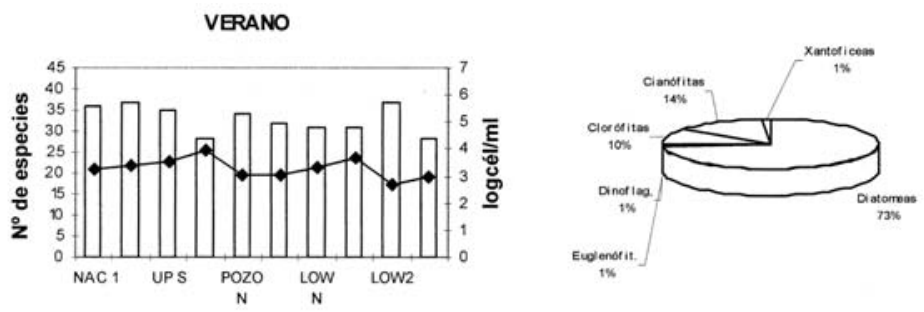

OTOÑO
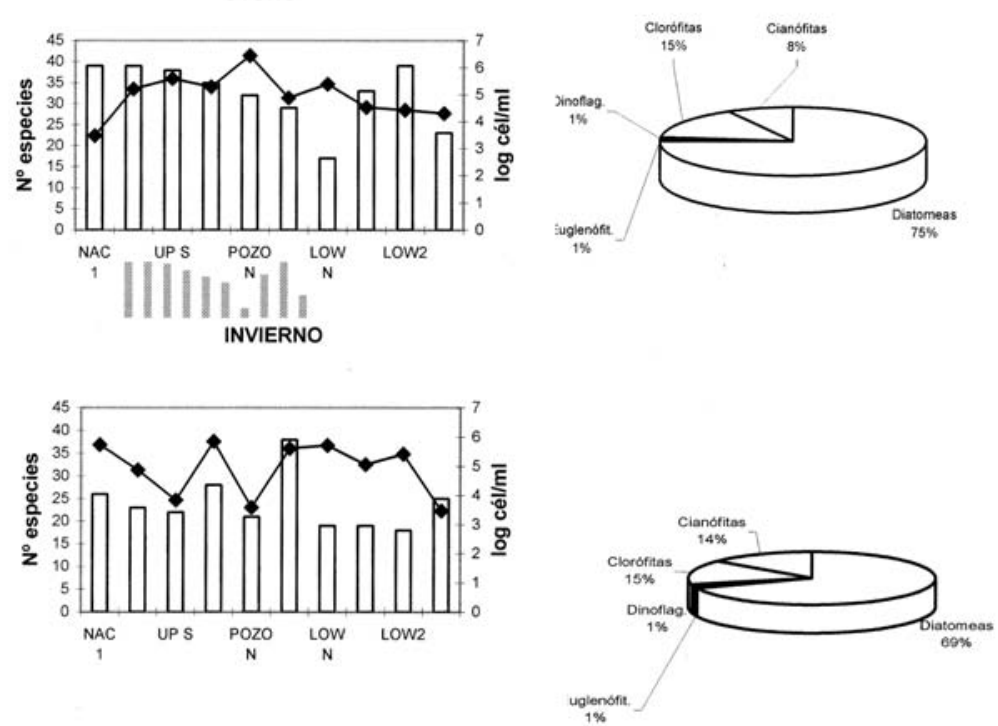

PRIMAVERA
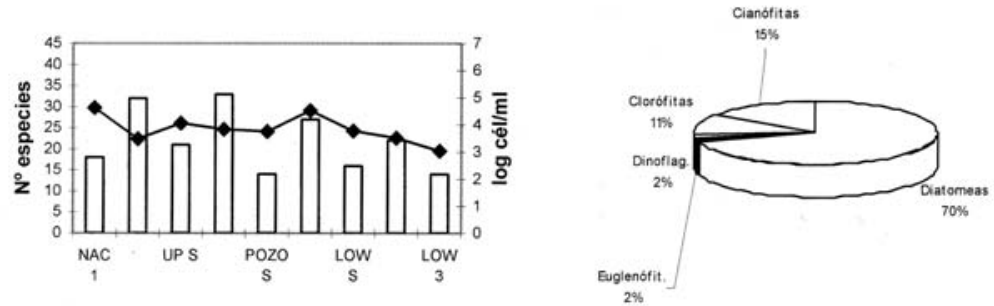

Figura 2. Número de especies, densidad estacional de fitoplancton (cel/ml) y proporción de grupos algales del Bañado de Crarilauquen en el periodo de estudio. Number of species, seasonal density of phytoplankton (cell/ml), and proportion of algal groups of the Bañado de Carilauquen during the study period. 
ca, Oscillatoria formosa, O. princeps, especies del género Anabaena y Pseudoanabaena y por las diatomeas Eunotia pectinalis y Nitzschia palea, principalmente en Nac 2.

\section{Fitobentos}

La diversidad promedio de la comunidad de algas bentónicas no presentó variaciones significativas a lo largo del año, con valores del índice de Shannon de 1.5. En la temporada estival la fracción bentónica estuvo dominada por diatomeas y los picos de abundancia se debieron a la proliferación de las poblaciones de Achnanthes exigua y Eunotia pectinalis. La densidad (celular por centímetro de superficie) correspondió a los valores menores del ciclo anual analizado con $10949 \mathrm{cel} / \mathrm{cm}^{2}$, (Fig. 3). En el otoño se observó la mayor densidad fitobentónica con un promedio de 4 millones de cel $/ \mathrm{cm}^{2}$. Rhopalodia gibberula se destaca por su abundancia. Esta especie ha sido registrada en otras localidades de la provincia como típica de otoño en ambientes lénticos (Peralta y Claps, 2000). Se destaca en esta temporada la dominancia de especies de Oscillatoria, Anabaena, Lyngbya limnetica y
Spirulina princeps en Nac 1, Low N y desembocadura del bañado. La diatomea Nitzschia palea se observó con alta densidad en Nac 1 y Nac 2 . En estos sitios se encuentran edificaciones domésticas precarias, lo que constituiría la principal fuente contaminante de las nacientes, además de la presencia del ganado doméstico asociado. En la temporada de invierno se observó un marcado incremento de especies de cianofitas, con alta abundancia de Oscillatoria formosa y Pseudoanabaena sp., principalmente en las nacientes, sector medio del bañado (Pozo N) y en Low N. Se destaca esta última estación de muestreo, la cual presentó el menor valor de diversidad en la temporada, con elevada densidad y dominancia de las mencionadas especies de cianofitas. El desarrollo de floraciones indican condiciones de elevada contaminación (Gómez, 1999), lo que podría deberse en este caso, a la presencia de hidrocarburo en el agua (11.8 mg/1). Asimismo, en primavera, en las nacientes y sector inferior del bañado (Low $\mathrm{S}$ y Low N) dominaron las especies de Oscillatoria, Peudoanabaena, A. torulosa, L. limnetica y $N$. palea. La densidad fue elevada, con 3702976 cel $/ \mathrm{cm}^{2}$ en promedio (Fig. 3).
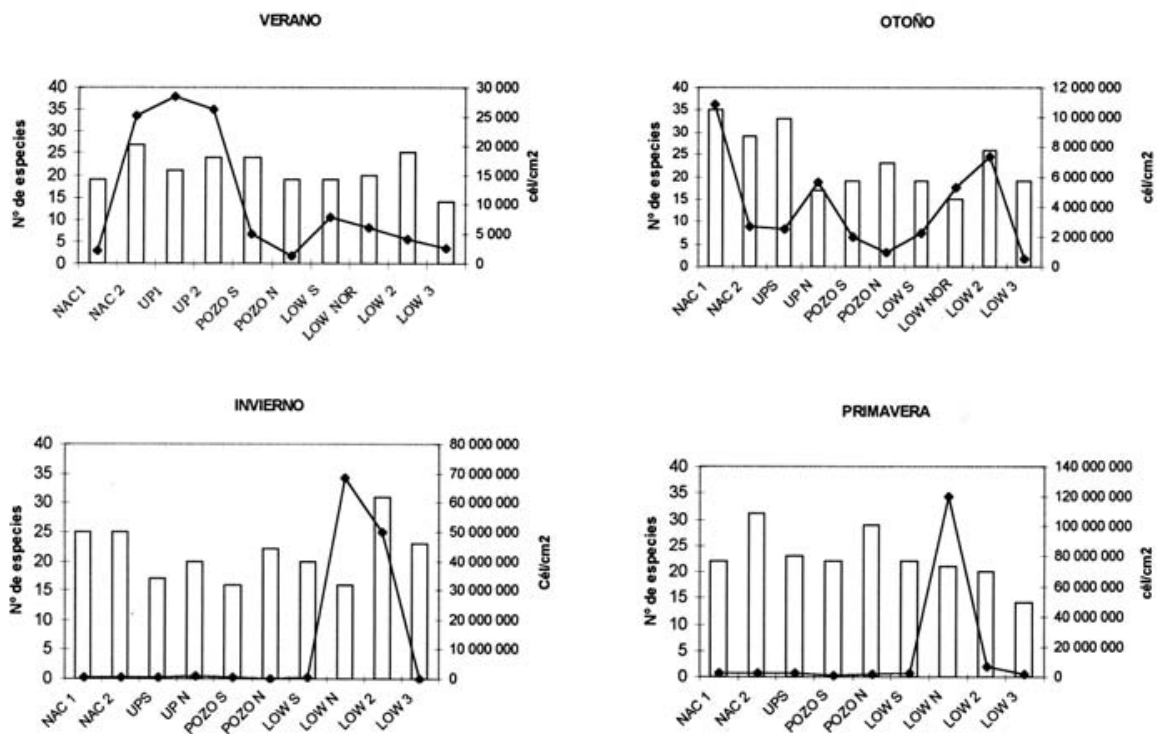

Figura 3. Riqueza de especies y densidad estacional de algas bentónicas (cél $/ \mathrm{cm}^{2}$ ), Bañado de Carilauquen. Species' richness and seasonal density of benthonic algae $\left(\mathrm{cell} / \mathrm{cm}^{2}\right)$, Bañado de Carilauquen. 
Tabla 4. Especies de zooplancton determinadas en el Bañado de Carilauquen. Zooplankton species determined in the Bañado de Carilauquen.

\begin{tabular}{|c|c|c|c|c|c|c|c|c|c|c|}
\hline & Nac 1 & Nac 2 & Up S & Up $\mathbf{N}$ & Pozo S & Pozo N & Low $S$ & Low $\mathbf{N}$ & Low 2 & Low 3 \\
\hline \multicolumn{11}{|l|}{ Tecamebas } \\
\hline Arcella hemisphaerica & IP & I & I & & & & $\mathrm{P}$ & & & \\
\hline Centropyxis aculeata (Ehrenberg) Stein & V O I P & V O I P & V O I P & VO I & V O IP & V O I P & V O I & V O IP & & $\mathrm{V}$ \\
\hline Cyclopyxis arcelloides (Nach De Flandre) & V O I & V I P & V O I & $\mathrm{V} \mathrm{O} \mathrm{I}$ & V O I P & V O I P & $\mathrm{V} \mathrm{O} \mathrm{I}$ & V O I & $\mathrm{O}$ & \\
\hline Difflugia elegans Penard & I & I & I & & I & I & I & O I & & \\
\hline Difflugia corona & & & & & $\mathrm{P}$ & $\mathrm{P}$ & & & & \\
\hline Difflugia gramen Penard & $\mathrm{O}$ & & O I P & I & O I & O I P & O I P & O I P & & \\
\hline Euglypha acanthophora (Ehrenberg) Perty & VOI & V O IP & $\mathrm{VOI}$ & V I & V O I P & V O I & V I & VOP & $\mathrm{O}$ & \\
\hline Protococurbitella sp. Gauthier-Lievre \& Thomas & $\mathrm{V}$ & & & & & & & & & \\
\hline \multicolumn{11}{|l|}{ Ciliados } \\
\hline Halteria sp. & & & & & & I & I & I & & \\
\hline Paramecium caudatum & IP & I & $\mathrm{P}$ & & $\mathrm{P}$ & $P$ & & & & \\
\hline Rhabdostyla sp. & $\mathrm{O}$ & & I P & & $\mathrm{V}$ & & $\mathrm{P}$ & I & $\mathrm{V}$ & \\
\hline \multicolumn{11}{|l|}{ Spirostomus sp. } \\
\hline Strombidium sp. & & I & & & & & & & & \\
\hline Vaginicola sp. & V I & V I & $\mathrm{V} \mathrm{O} \mathrm{I}$ & $\mathrm{VO}$ & $\mathrm{V}$ & & $\mathrm{V}$ & $\mathrm{V}$ & V P & V O I P \\
\hline \multicolumn{11}{|l|}{ Rotiferos } \\
\hline Bdeloideo & OIP & $\mathrm{VOI}$ & V I & $\mathrm{VO}$ & V I & V O IP & VO I & $\mathrm{V} \mathrm{O} \mathrm{I}$ & $\mathrm{O}$ & $\mathrm{VO}$ \\
\hline Brachionus caudatus & & & & & & & & I & & \\
\hline Brachionus quadridentatus (Hermann) & & & & & & & & $\mathrm{V}$ & $\mathrm{V}$ & $\mathrm{V}$ \\
\hline Colurella hinderbergi & & & & & & $\mathrm{P}$ & & & & \\
\hline Euchlanis dilatata (Ehrenberg) & $\mathrm{VO}$ & $\mathrm{VO}$ & & $\mathrm{O}$ & $\mathrm{V}$ & $\mathrm{VO}$ & $\mathrm{VO}$ & $\mathrm{VO}$ & $\mathrm{V}$ & \\
\hline Keratella quadrata & & & & & & & & & I & I P \\
\hline Keratella sp. (Bory St. Vicent) & & & & & & $\mathrm{V}$ & & & & \\
\hline Lecane bulla (Gosse) & $\mathrm{VO}$ & $\mathrm{VO}$ & V O I & V O I & V I & $\mathrm{V}$ & V O I & V O I & $\mathrm{V}$ & V O I \\
\hline Lecane horneami & $\mathrm{P}$ & & & & & & & & & \\
\hline Lecane sp. & & & & & I & & & & & \\
\hline Lepadella ovalis (Müller) & V O I & V O I & V I & V O I & V O I & V I & V I & $\mathrm{V} \mathrm{O}$ & $\mathrm{O}$ & $\mathrm{O}$ \\
\hline Macrochaetus subquadratus Perty & & & & & & $\mathrm{O}$ & $\mathrm{V}$ & & & \\
\hline Monommata sp. & & & & & & & & O I & & \\
\hline Notholca acuminata (Ehrenberg) & & & $\mathrm{V}$ & & & & & & & \\
\hline Notholca striata & & O I & $\mathrm{I}$ & I & I & I & I & I P & $\mathrm{P}$ & $\mathrm{P}$ \\
\hline Platyas sp. & & & & & & I & & & & \\
\hline Squartinella sp. (Bory St. Vicent) & & & & & $\mathrm{O}$ & & $\mathrm{V}$ & $\mathrm{V}$ & & \\
\hline Trichocerca iernis (Gosse) & $\mathrm{VO}$ & $\mathrm{V}$ & $\mathrm{P}$ & & $\mathrm{O}$ & $\mathrm{O}$ & $\mathrm{V}$ & $\mathrm{O}$ & & \\
\hline Trichocerca sp. & & & I & I & & & & & & \\
\hline
\end{tabular}

\begin{tabular}{|c|c|c|c|c|c|c|c|c|c|c|}
\hline $\begin{array}{l}\text { Nematodos } \\
\text { no identificado }\end{array}$ & $\mathrm{V}$ & V P & & V I & V O I & O I & V I & $\mathrm{V}$ & & \\
\hline \multicolumn{11}{|l|}{ Ostracodos } \\
\hline Ostracodo sp. 1 & $\mathrm{VO}$ & V O I & V O I & V O I & V O P & V O I & V I & $\mathrm{V}$ & $\mathrm{VO}$ & V O I \\
\hline Ostracodo sp. 2 & V O I P & V O I P & V O P & V P & $\mathrm{OP}$ & V I P & IP & $\mathrm{O}$ & & $\mathrm{O}$ \\
\hline Ostracodo sp. 3 & & & & & V O & $\mathrm{V}$ & V I & V & $\mathrm{V}$ & \\
\hline Ostracodo sp. 4 & & I & & $\mathrm{O}$ & $\mathrm{VO}$ & V I & I & & & \\
\hline \multicolumn{11}{|l|}{ Copepodos } \\
\hline Paracyclops fimbriatus (Fischer) & V O I & $\mathrm{VO}$ & & $\mathrm{VO}$ & $\mathrm{VO}$ & $\mathrm{VO}$ & I & I & V O P & \\
\hline Boeckella vergi & & & & & & & & & $\mathrm{P}$ & $\mathrm{P}$ \\
\hline \multicolumn{11}{|l|}{ Cladoceros } \\
\hline Camptocercus sp. & $\mathrm{V}$ & & & & & & & & & \\
\hline Chydorus sphaericus (Müller) & $\mathrm{V}$ & $\mathrm{V}$ & $\mathrm{P}$ & $\mathrm{VO}$ & $P$ & V I & V P & & & \\
\hline Macrothrix hirsuticornis Norman y Brady & $\mathrm{V}$ & & $\mathrm{V}$ & $\mathrm{V}$ & & & $\mathrm{VO}$ & $\mathrm{O}$ & & \\
\hline Simocephalus vetulus & & $\mathrm{V}$ & & $\mathrm{V}$ & & & $\mathrm{V}$ & & & \\
\hline nauplii & V O I & V O I & V I & V O I & $\mathrm{VO}$ & $\mathrm{O}$ & V O I & V O I & V O P & V O I P \\
\hline
\end{tabular}




\section{Zooplancton}

En el zooplancton del Bañado Carilauquen se identificaron un total de 44 taxa: 19 rotíferos; 8 amebas tecadas; 6 ciliados; 4 ostrácodos; 4 cladóceros; 2 copépodos y un nemátodo (Tabla 4). Esta comunidad se caracterizó por la presencia de especies euplanctónicas, ticoplanctónicas y otras netamente bentónicas. Se observó variabilidad tanto estacional como espacial en lo referente a dominancia de grupos, composición y abundancia específica.
Las menores densidades zooplanctónicas (ind/l) se hallaron en las nacientes, en la zona de desembocadura y Laguna de Llancanelo, mientras que los mayores valores se detectaron en las zonas medias de la cuenca. Las amebas tecadas fueron las responsables de los picos de densidad (Fig. 4). El menor número de especies por sitio se detectó en la temporada de primavera, los mayores en verano y otoño, y valores medios en invierno (Fig. 4). Los sitios Low 2 y Low 3 poseen bajos valores promedio en densidad y riqueza de especies (Low 2: 468 ind/1, 6 espe-
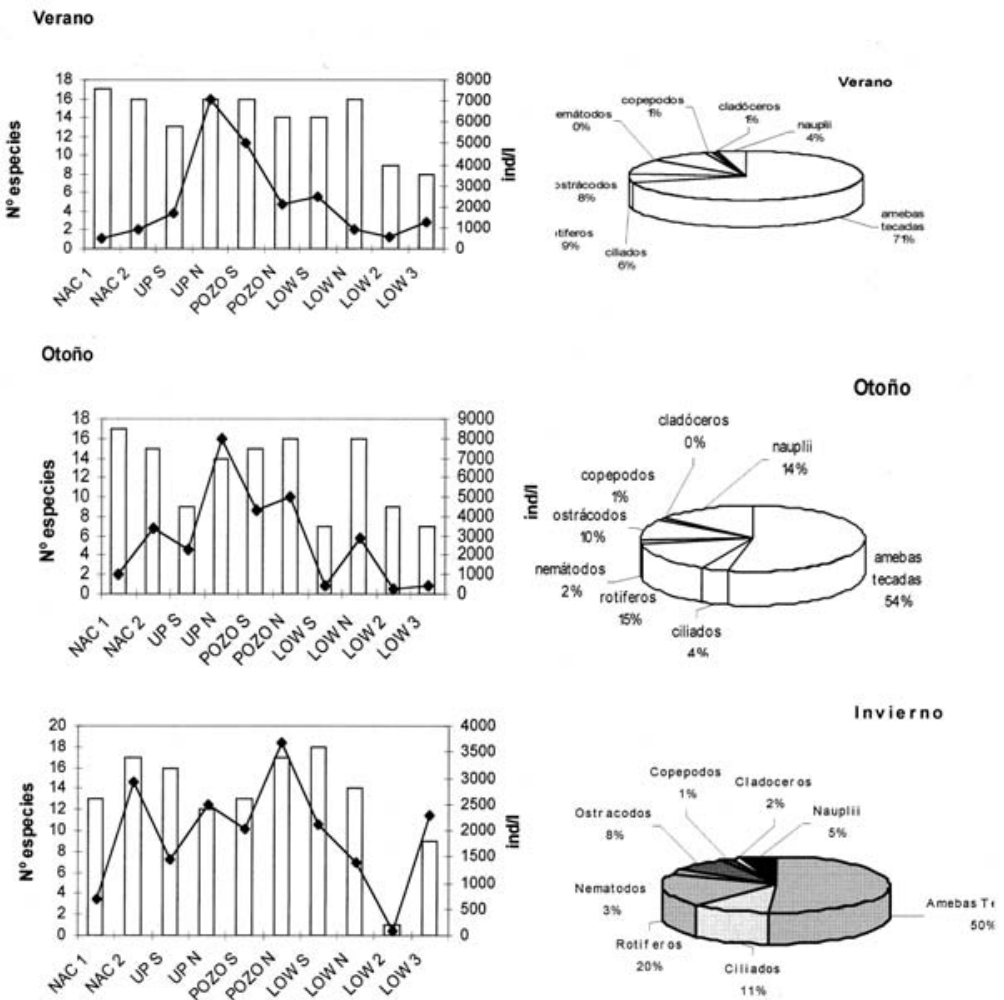

Primavera
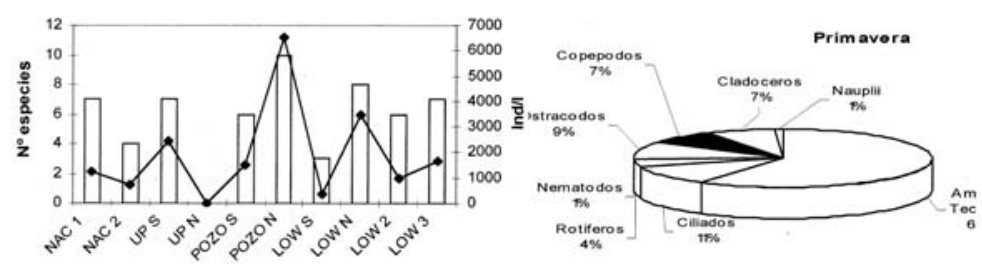

Figura 4. Variación estacional y espacial del número de especies, abundancia de organismos zooplanctónicos (ind/ml) y proporción de grupos de zooplancton presentes en el Bañado de Carilauquen. Seasonal and spatial variation of the number of species, abundance of zooplanktonic organisms (ind/ml), and proportion of zooplankton groups present in the Bañado de Carilauquen. 
cies; Low 3: 1398 ind/1, 11 especies). Estos valores pueden atribuirse a la elevada concentración de iones presentes en este sector de la cuenca. La salinidad es un factor importante que afecta la distribución de los invertebrados acuáticos en general, influyendo tanto en la riqueza de especies como en la composición de la comunidad (Halse et al., 1998).

Por otro lado, estudios realizados en ecosistemas de humedales sugieren que las comunidades de zooplancton están estructuradas principalmente por componentes bióticos, como los efectos de la vegetación litoral (macrófitas) que sirven de refugio (Wetzel, 1981; Timms and Moss, 1984; Cole, 1988; Hansen and Jeppesen, 1992; Schriver et al., 1995) y disponibilidad de alimento (Loughedd et al., 1998). En este sentido cabe destacar el muy bajo porcentaje de cobertura vegetal existente en las zonas de la desembocadura y laguna de Llancanelo (Peralta y Fuentes, 2001).

En la figura 4 se representa la proporción de los distintos grupos del zooplancton, observándose que las amebas tecadas fueron el grupo dominante en las 4 estaciones estudiadas. Los sitios de muestreo presentaron diferencias en cuanto a los grupos de zooplancton dominantes. El zooplancton de la zona de nacientes estuvo mayormente representado por amebas tecadas en Nac 1, rotíferos en Nac 2 y ciliados en invierno en ambos sitios. El ciliado más abundante fue Paramecium caudatum, especie típica de ambientes con elevada carga orgánica (Bick, 1972), en sitios donde los niveles de reducción de oxígeno son altos y las poblaciones de bacterias tienden a ser muy abundantes (Wetzel, 1981). La presencia de rotíferos en ambos sitios y principalmente en NAC 2, puede relacionarse a la vegetación existente. Se encontraron grandes masas de macroalgas perifíticas representadas por Zygnema pectinatum y Spyrogyra mirabili) que sirven de hábitat para rotíferos que viven en estrecha asociación con el perifiton, como lo son Trichocerca iernis y Lepadella ovalis.

En los sectores medios del Bañado (Up N, Up $\mathrm{S}$, Pozo $\mathrm{N}$ y Pozo S) predominaron las amebas tecadas, las cuales pueden ser buenas indicadoras de un número importante de perturbaciones de origen antropogénico (Patterson, et al., 2000). En ambientes estresados (aguas salobres, altos niveles de contaminantes, contaminación orgánica) las comunidades de amebas tecadas muestran una baja diversidad, dominadas por Centropyxis aculeata y Arcella vulgaris (Dalby, et al., 2000). Los sitios mencionados poseen ciertas características que pueden resultar estresantes. Las condiciones altamente fluctuantes, la elevada temperatura y evaporación del agua en la temporada estival favorecen la dominancia de especies caracterizadas por su amplia distribución y tolerancia a diferentes condiciones ambientales (Hutchinson, 1967).

En las zonas de la desembocadura y laguna de Llancanelo se observó estacionalidad en la composición taxonómica de rotíferos. Brachionus quadridentatus se detectó en estos sitios exclusivamente en verano, lo que coincide con otros trabajos donde esta especie fue exclusiva de la temporada estival (Guisande et al., 1988). Es considerada una especie heleoplanctonica ya que prefiere ambientes poco profundos (Pejler et al., 1989) y es común en ambientes salinos (Bick, 1972). En otoño, en LOW 3, la fauna de rotíferos estuvo representada por las especies Lecane bulla y Lepadella ovalis, especies de hábitos perifíticos. En la temporada de invierno, en Low 2 y Low 3 se detectarona a los rotíferos estenoternos del frío Notholca striata y Keratella quadrata. (Pejler, 1975). Los copépodos estuvieron representados por dos especies planctónicas: Paracyclops fimbriatus y Boeckella bergi. Paracyclops fimbriatus estuvo presente en mayores abundancias en las temporadas de verano y otoño, principalmente en las estaciones aguas arriba. Boeckella bergi, fue hallada en la temporada de primavera, en los sitios LOW 2 y LOW 3. Se identificaron cuatro especies de cladóceros en bajas densidades, a excepción de Chydorus sphaericus en primavera.

\section{DISCUSIÓN}

Muchas de las especies identificadas son conocidas como típicas de ambientes eutrofizados: Diatoma vulgare, Melosira varians, Navicula cryptocephala, 
Pinnularia gibba, Pinnularia maior, Rhoicosphaenia curvata, Rhopalodia gibba, Stauroneis phoenicenteron y Synedra acus. Además de especies de géneros de cianofitas como Oscillatoria, Lyngbya, Anabaena y Pseudoanabaena y el ciliado $P$. caudatum. Estas especies se han observado con alta frecuencia en los sitios correspondientes a las nacientes (Nac 1 y Nac 2), sector medio del bañado, $($ Low $\mathrm{N})$ y desembocadura.

El análisis destinado a la caracterización del bañado según el estado de saprobiedad, calculado por el índice de Sladecek para las comunidades zooplanctónicas y algales, determina la característica polisapróbica (valores entre 3 y 4 ) del bañado de Carilauquen. Es decir intensa degradación de materia orgánica y/o putrefacción.

Asimismo, los resultados reflejan la alta productividad del ambiente, común en humedales. El Bañado de Carilauquen presenta densa cobertura tanto de algas bentónicas como de macrófitas acuáticas, especialmente de Scirpus californicus y Chara vulgaris. Estas especies presentan estacionalidad marcada, por lo que en temporadas de bajas temperaturas el aporte de material vegetal al cuerpo de agua es intenso. Se debe sumar, el aporte de materia orgánica de origen animal, proveniente de la intensa carga ganadera caprina y vacuna en la zona.

Como indicadoras de la baja calidad del agua, se suma la presencia en alta densidad de las diatomeas N. palea, Gomphonema parvulum y Navicula goppertiana. Éstas últimas son conocidas como taxa tolerantes a la contaminación orgánica, (Lange Bertalot, 1979; Lobo et al., 1996) y en general indican condiciones polisapróbicas.

\section{CONCLUSIONES}

Se observa un marcado gradiente en los valores de conductividad desde las nacientes $(959 \mu \mathrm{S}$ $\mathrm{cm}^{-1}$ promedio) a la desembocadura (10 $810 \mu \mathrm{S}$ $\mathrm{cm}^{-1}$ promedio). Este gradiente está determinado por el aumento en la concentración (mg/l) de los iones sulfato, bicarbonato, cloro, sodio, potasio y magnesio, definiendo tres secciones: superior correspondiente a las nacientes (Nac 1 y Nac 2); media : Up S, Up N, Pozo S, Pozo N, Low $\mathrm{S}$ y Low N; inferior : desembocadura (Low 2) y Laguna de Llancanelo (Low 3).

Entre los componentes biológicos lanctónicos y bentónicos considerados, se observa una respuesta al patrón espacial mencionado. Las zonas donde la salinidad fue alta fueron colonizadas por especies de diatomeas características de ambientes salobres y/o marinos de los géneros Amphora, Amphiprora, Campylodiscus, Hyalodiscus. Entre los rotíferos tolerantes adaptados a altas concentraciones de sal se destacaron B. quadridentatus, L. bulla, Euchlanis dilatata, L. ovalis, Notholca striata, Keratella quadrata y el copépodo B. bergi.

Los grupos algales dominantes identificados en el bañado de Carilauquen son comunes a los humedales de agua dulce ricos en nutrientes de regiones templadas: diatomeas pennadas (géneros Achnanthes, Cocconeis, Cymbella, Epithemia, Fragilaria, Gomphonema, Navicula y Nitzschia) algas verdes filamentosas (géneros Spyrogyra, Oedogonium, Uronema y Zygnema) y cianofitas (Anabaena, Lyngbya, Oscillatoria, Pseudoanabaena), (Stevenson et al., 1996).

Los estudios referidos a las comunidades planctónicas y bentónicas algales mostraron a las diatomeas pennadas como el grupo algal dominante en todas las estaciones del año y en todos los sitios de muestreo. Las cianofitas y clorofitas fueron co-dominantes.

En cuanto al zooplancton, las amebas tecadas fueron el grupo proporcionalmente más abundante en todas las estaciones, seguidos en importancia por los rotíferos, ostrácodos y ciliados. Los copépodos y cladóceros fueron grupos proporcionalmente menos abundantes.

Debido a la baja profundidad del bañado y a los fuertes vientos reinantes en la zona, la fracción planctónica estuvo comprendida por numerosas especies ticoplanctónicas, y sólo 2 especies planctónicas propiamente dichas (Fragilaria ulna y Asterionella formosa).

En relación con el zooplancton, se detectó la presencia de especies euplanctónicas, ticoplanctónicas y bentónicas.

La diversidad algal planctónica presentó valores altos en la temporada de verano y bajos en 
invierno. Mientras que la fracción bentónica presentó valores menores en primavera.

La presencia de hidrocarburo en Low $\mathrm{N}$ y Low 2 podría ser la causa de la disminución de la diversidad planctónica - bentónica, y proliferación de algas filamentosas correspondientes al grupo de cianofitas. Entre ellas se destacan Anabaena torulosa, A. inaequalis, L. limnetica y especies del género Oscillatoria.

La mayor riqueza de especies de organismos zooplanctónicos fue observada en la temporada de verano, seguida por invierno y otoño. Mientras que el menor número de especies se detectó en la primavera.

En todas las temporadas analizadas, los picos de dominancia algal fueron dados por algas filamentosas del grupo de cianofitas, a excepción del verano donde la dominancia estuvo dada por las diatomeas Achnanthes exigua y E. pectinalis. Mientras que entre los organismos zooplanctónicos los picos de abundancia, en la mayoría de los casos, fueron producidos por el grupo de las amebas tecadas (principalmente $C$. arcelloides y $C$. aculeata). Los rotíferos presentaron picos de abundancia en invierno en los sitios Nac 1, Up S, Pozo S y Low S. Los ciliados incrementaron su densidad en invierno y primavera en los sitios Nac 1 y Nac 2.

Tanto la proporción de las especies indicadoras (N. palea, G. parvulum, N. goppertiana y $P$. caudatum, entre otras) como el índice calculado sobre la comunidad fito y zooplanctónica, definen al bañado como polisapróbico con elevada concentración de polución orgánica. El Bañado de Carilauquen no recibe descarga de actividad industrial ni agrícola. Por lo tanto, las únicas fuentes de aporte lo constituyen las macrófitas, algas y desechos fisiológicos de origen animal. Se destaca el impacto que reciben las nacientes por actividad humana y ganadera.

\section{AGRADECIMIENTOS}

Agradecemos a: Fundación CRICYT; Dirección de Recursos Naturales, Delegación Malargüe, especialmente a su cuerpo de Guardaparques;
Marcelo Espinola por la colaboración recibida en los trabajos de campo; Dra. Cristina Claps por la lectura crítica del manuscrito. A los revisores anónimos por las sugerencias realizadas.

\section{BIBLIOGRAFIA}

APHA. 1995. Standard methods for the examination of water and wastewater. 17th ed. APHA, Washington, D.C. 1550 pp.

BICK, H. 1972. Ciliated Protozoa. An illustrated guide to the species used as biological indicators in freshwater biology. World Health Organization. Geneve. 198 pp.

BLENDINGUER, P. 2001. Estudio de Base Cero, Bañado de Carilauquen. Informe Final Aves. Fundación Cricyt, 47pp.

COLE, G. A. 1988. Manual de Limnología.. Editorial Hemisférico Sur S. A., Buenos Aires. 405 pp.

DALBY, A.P., A. KUMAR, J. M. MOORE, \& R. T. PATTERSON. 2000. Preliminary assessment of arcellaceans (Thecamoebians) as limnological indicators in Tropical Lake Sentani, Irian Jaya, Indonesia. Journal of Foraminiferal Research., 30: 135-142.

DESCY, J. P. \& M. COSTE. 1990. Utilisation des diatomées benthiques pour l'evaluation de la qualité des eaux courantes. Contrat CEEB-71-23. Rapport final. Cemagref. $60 \mathrm{pp}$.

GÓMEZ, N. 1999. Diatomeas Bentónicas como indicadoras de la calidad del agua en sistemas lóticos de la Llanura Pampeana. En: Bentos regional argentino. Las comunidades lóticas bioindicadoras. D. H. Dipersa (ed.). Ed: 1-28 Cient. Amer. La Plata.

GUISANDE, C. \& J. TOJA. 1988. The dynamics of various species of the genus Brachionus (Rotaria) in the Guadalquivir River. Arch. Hydrobiol., 112: 579-595.

HALSE, S. A., R. J. SHIEL \& W. D. WILLIAMS. 1998. Aquatic invertebrates of Lake Gregory, northwestern Australia, in relation to salinity and ionic composition. Hydrologia, 381: 15-29.

HANSEN A. M. \& E. JEPPESEN. 1992. Changes in abundance and composition of cyclopoid copepods following fish manipulation in eutrophic Lake Voeng. Denmark. Freshwat. Biol., 28: 183-193.

HUTCHINSON, G. E. 1967. A Treatise of Limnology. Geography, Physics and Chemistry. John Wiley \& Sons, Toronto. 1115 pp. 
IGLESIAS, G. J. \& A. A. PÉREZ. 1998. Patagonia. En: Los humedales de la Argentina: clasificación, situación actual, conservación y legislación. Canevari, P., D. E. Blanco, E. H. Bucher, G. Castro \& I. Davidson (eds.): 117-135. Wetlands International Publ. 46, Buenos Aires.

LANGE BERTALOT, H. 1979. Pollution and tolerance of diatoms as criterion of water quality estimation. Nova Hedwigia, 64: 285-304.

LOBO, E., V. L. CALLEGARO, M. A. OLIVEIRA, S. SALOMONI, S. SCHULER \& K. ASAI. 1996. Pollution Tolerant Diatoms from Lotic System in the Jacuí Basin, Rio Grande do Sul, Brazil. Iheringia (Ser. Bot. Porto Alegre), 47: 45-72.

LOUHEED, V. L. \& P. CHOW-FRASER. 1998. Factors that regulate the zooplankton community structure of a turbid, hypereutrophic Great Lake wetland. Can. J. Fish Aquat. Sci., 55: 150-161.

OSTERA, H. A. 2001. Estudio Geoquímico Base Cero, Bañado de Carilauquen. Hidrocarburos en suelos. Hidrocarburos en gas de suelo. Hidrocarburos en agua. Hidrogeoquímica de superficie. Informe verano 2001. Fundación Cricyt. 98 pp.

PAERL, H. 1988. Growh and reproductive strategies of freshwater Blue-green algae (Cyanobacteria).

En: Growh and reproductive strategies of Freswater Phytoplankton. Ed. Graig. Sandgren.: 261-316. Univ. of Wisconsin, Milwaukee.

PATTERSON, R. T. \& A. KUMAR. 2000. Use of arcellacea to gauge levels of pollution and remediatin of industrially lakes. Environmental Micropaleontology, 15: 257-278.
PEJLER, B. 1975. Taxonomical and ecological studies on planktonic Rotaria from central Sweden. Lapland. K.V.A. Handl., 6 (5): 1-68.

PEJLER, B. \& B. BERZINS. 1989. On choice of substrate and habitat in brachionid rotifers. In: Ricci, C., T. Snell \& C. King (eds). Rotifers Symposium V. Hydrobiologia, 186/187: 137-144.

PERALTA, P. \& C. CLAPS. 2000. Plankton of a high mountain lake (High Los Andes Cordillera, Central west of Argentina): a approach of its seasonal variations. Verh. Inter. Verein. Limnol., 28: 1036-1040

PERALTA. P. \& V. FUENTES. 2001. Estudio Base Cero Carilauquen. Informe Final Vegetación Litoral. Fundación Cricyt. 24 pp.

SCHRIVER, P. B., J. BOGESTRAND, J. JEPPESEN \& M. SONDERGAARD. 1995. Impact of submerged macrophytes on fish- zooplankton-phytoplankton interactions: large-scale enclosure experiments in a shallow eutrophic lake. Freswat. Biol., 33: 255-270.

SOSA, H., F. VIDELA \& S. PUIG. 1989. Marcación de límites biológicos del sector No.O. de la Reserva Provincial Laguna de Llancanelo. Informe interno Dirección de Recursos Renovables, Provincia de Mendoza. 20 pp.

TIMMS, R. M. \& B. MOSS. 1984. Prevention of growth of potentially dense phytoplankton populations by zooplankton grazing, in the presence of zooplanktivorous fish in a shallow wetland ecosystem. Limnol. Oceanogr., 29: 472-486.

WETZEL, R. G. 1981. Limnología. Ediciones Omega. Barcelona. 679 pp. 
